\title{
Generalized "Stigma": Evidence for Devaluation-by-Inhibition Hypothesis from Implicit Learning
}

\author{
Haotian $\mathrm{Zhou}^{1,2, *,}$ Lulu Wan ${ }^{1,2, *}$, and Xiaolan $\mathrm{Fu}^{1, * *}$ \\ ${ }^{1}$ State Key Laboratory of Brain and Cognitive Science, \\ Institute of Psychology, Chinese Academy of Sciences, \\ Beijing 100101, China \\ \{zhouht, wanll, fuxl\} @psych.ac.cn \\ ${ }^{2}$ Graduate School, Chinese Academy of Science, Beijing 100039, China
}

\begin{abstract}
Recently, a new fundamental discovery has been made of the relationship between attentional system and affective system of human brain, giving rise to the devaluation-by-inhibition hypothesis. It is shown that selective attention has an affective impact on an otherwise emotionally bland stimulus. Particularly, if a neutral stimulus was inhibited by selective attention in a prior task, it would be valued less in a subsequent affective evaluation task than it would otherwise have been. In the present study, we extend this line of research on the affective consequence of attention and demonstrate that prior attentional states (attended or inhibited) associated with a group of neutral stimuli (character strings) can even influence subsequent preference judgment about previously-unseen stimuli if these new stimuli share certain basic features (e.g., follow the same rule) with those encountered in a previous stage.
\end{abstract}

Keywords: Affective evaluation, attentional inhibition, implicit learning, artificial grammar learning.

\section{Introduction}

People from all walks of life would agree that emotion is an integral part of human existence [16]. The important role played by emotion in our daily life is wide-ranging and cannot be overestimated. During the past several decades, emotion as an academic subject has been studied intensely by psychologists, computer scientist, biologists, and the likes. According to Cacioppo and Gardner [4], one of the major focuses of the scientific endeavor to unravel the mystery of emotion is centered on the interaction between emotion and cognition. Considerable effort goes into addressing issues such as how emotion influences decision-making, memory and creativity [1][2][13]. Nevertheless, much of the insight we have gained into the interaction

\footnotetext{
* These two authors contributed equally to this paper.

** Corresponding author.
} 
between emotion and cognition comes from the studies dealing with the interplay between emotion and attention.

\subsection{Emotion Influences Attention}

Given the significance of both attention and emotion to the survival of human race, it is not surprising that substantial evidence has been accumulated showing that emotional state exert great impact on attentional process. Research has demonstrated that affective stimuli (e.g. faces with expressions, sexually-relevant pictures, et al.) attract attention compared with neutral or meaningless stimuli. For instance, Hao and colleagues showed that compared with neutral target, emotionally-salient target is located faster amongst multiple neutral distracters [8]. Other studies have found that the scope of attention can be modulated by the subjective emotional states. For example, in Rowe's recent experiment [13], participants in negative mood were less likely to be influenced by the interference from nearby distracters than their counterparts in positive mood. Moreover, some researchers have found that emotional disorders can profoundly alter one's selective attention [10]. In fact, one would be hard-pressed to find one study which contradicts the general finding that attention is subject to the influence of emotion.

\subsection{Attention Influences Emotion}

A number of functional imaging studies have discovered that the neural substrates underlying attention and emotion are not only connected but also, in some cases, they reside in the same brain regions (e.g. [3]). Thus, one should expect a reciprocal relationship between attention and emotion. However, for quite a long period, the situation is lopsided in terms that much of the effort has been devoted to deciphering the possible influence of emotion on attention while little has been done about the flipping side of the question-how attention affects emotion [6].

Recently, this long over-looked question has been taken up by Fenske and Raymond. In a series of studies [7][11], they made an important discovery that selective attention - the process of selecting a subset of stimuli from amongst many potentially available ones to focus on-is capable of swaying affective judgment of stimuli in a systematic way. Specifically, they propose a devaluation-by-inhibition hypothesis which asserts that the attentional state (attended or inhibited) assigned to a stimulus when it is first encountered can influence subsequent affective judgment of the same stimulus [7]. For example, if an emotionally bland stimulus served as distracter in the initial task (hence must be inhibited), it will be rated less cheerful than its target counterpart, which is neutral in nature, in a following evaluation task. In other words, attentional inhibition can depreciate the value of a given stimulus.

\subsection{Stigmatized Representation}

In their conceptualization, Raymond and colleagues suggest that the attentional inhibition associated with a stimulus functions very much like a stigma and is attached to the representation of the stimulus [11]. Therefore, whenever the representation is activated again, this inhibition-based stigma is also invoked and devalues the stimulus automatically. Such a stigma analogy is reminiscent of the 
activation of social stereotype from social psychology. Yet, in social stereotype, the stigma is attached to the representation of a group on the basis of certain common traits possessed by group members, whereas in Raymond and Fenske's studies, the stigma is attached to the representation of each individual exemplar [6]. A natural question to ask, hence, is: Can the inhibition-based stigma proposed by Raymond and Fenske attach to a group-level representation so that a stimulus, which in itself has not been subject to attentional inhibition but nevertheless shares certain commonalities with those inhibited stimuli, will be devalued once encountered in an evaluation task.

As a matter of fact, abstraction and generalization are important coping strategies for us to manage and organize the huge amount of information stored in our brains so that we will not be overwhelmed. It is clear that if the number of stimuli being inhibited exceeds certain numbers and all the stimuli share certain common diagnostic traits, then it is far more efficient to store a single inhibition-based stigma with an abstracted representation of the group than to store each individual stimulus along with their associated stigma. For example, if a person has some unpleasant encounter with a dog, it is very likely he will develop some aversion toward not that specific dog but dogs as a group.

In the present study, we seek to examine whether the devaluating effect of attentional inhibition can be spread to previously unseen stimuli which share certain diagnostic features with those inhibited stimuli.

\section{Method}

In this experiment, a variant of the artificial grammar learning (AGL) paradigm devised by Tanaka et al. [17] was adopted to address the research question (see section 2.3). AGL is a classical paradigm for the investigation of implicit learning [12]. A typical AGL procedure comprises two stages [5]. During the first stage (training stage), participants are required to memorize (i.e. rote learning) some character strings all of which follow the same rule (or grammar); then in the second stage (testing stage), they are required to classify whether the test items (unseen in the training stage) follow the same rule as those appeared in the first stage. Past studies using AGL have repeatedly shown that people can abstract the common complex rule underlying all the memorized items independent of conscious attempts to decipher such a rule and largely in the absence of explicit knowledge of what has been acquired [15]. Therefore, AGL provide us with the basic rationale for exploring the possibility that the affective consequence of attentional inhibition can be spread to new stimuli from the same family as those being inhibited. In specific, supposing that participants can acquire the rules underlying both the attended and inhibited strings in the training stage, we can test our hypothesis by examining whether the affective value of an unseen strings would be affected by its membership (i.e. to which rulesattended or inhibited - this new stimulus conforms) in a fashion as would be predicted from devaluation-by-inhibition hypothesis.

\subsection{Participants}

26 participants (6 males, 20 females) were recruited from the China Agriculture University. Participation was voluntary and all participants were reimbursed. They 
were naive to the experimental hypothesis and have normal or corrected-to-normal vision.

\subsection{Apparatus and Stimuli}

The experiment was conducted with a Pentium-IV computer connected to a 17 -inch monitor. E-Prime 1.2 was used to control the stimuli presentation and response recording [14]. Participants were seated about $60 \mathrm{~cm}$ from the monitor. All stimuli appeared on a uniform $50 \%$-gray field.

Strings of five to nine characters in length were generated according to one of the three different artificial grammars, Grammar D (GD), Grammar V (GV), and Grammar K (GK). GD was used by Dienes et al. [5], GV by Vokey et al. [18], and GK was a modified version of that used by Knowlton et al. [9]. To ensure that the strings from each grammar were comparable at the perceptual level, all three grammars consist of the same letter set, M, R, T, V, and X.

A total of 39 non-repeating GD strings were generated, of which 15 were used in training stage and the remainders in evaluation stage. This was the same case with GV strings. In addition, a total of 48 non-repeating strings were generated from GK, but were used solely in the evaluation stage.

\subsection{Design and Procedure}

In the training stage (Fig. 1A), each trial began with an 800-ms fixation point and was followed by the presentation of two character strings of the same length with one from GD and the other from GV. The two partially-overlapped strings of different colors (black and white) were presented simultaneously. The misalignment of the two strings was done in such a way that, in order to read one string of the pair correctly, the participants had to inhibit the interference from the other. The participants were instructed to type the string of the cued position (front or back) into the computer as fast as possible and then pressed the "Enter" key to proceed to next trial. The cue is a Chinese character meaning either front or back and displayed on the left of the string pair. Both the cue and the string pair would stay on screen until the onset of next trial. If participants submit a copy with error, the program would notice them to type again until the correct one was submitted. There were eight blocks in the training stage. Each block comprises 15 trials. Each of the 15 different GV-GD string pairs was presented once in each of the eight blocks with the position being determined randomly at the onset of each block.

Unbeknownst to the participants, throughout the whole stage, the strings to be typed were all conform to GD. Yet, their color (black or white) and position (front or back) were counterbalanced across the eight blocks.

In the evaluation stage (Fig. 1B), participants were instructed to complete a preference judgment task. In each trial, two strings of equal length were presented simultaneously and participants were required to indicate which one of the two they liked better by click on corresponding buttons on the screen. That is, if one prefers the string in the upper position, one should click the "U (up)" button, and if the one favors the string in the lower position, one should click the " $\mathrm{D}$ (down)" button. There were 48 trials in the evaluation stage. 
Training sequence

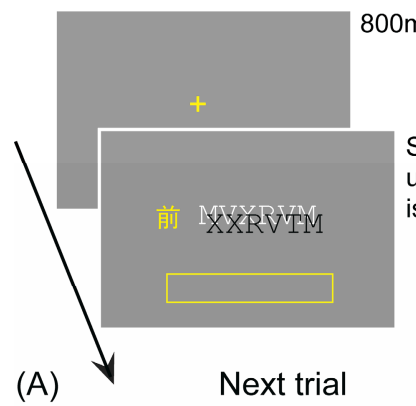

\section{Evaluation sequence}

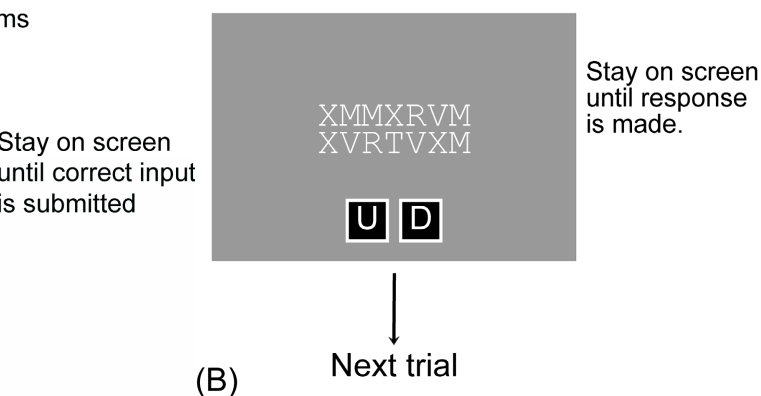

Fig. 1. (A) Time sequence of a single trial in training stage. The Chinese character on the left of the string pair indicates which string to copy. If the character is “前 (meaning front)", participants need to type in the string in the foreground. On the other hand, if it is "后 (meaning back)", participants need to type in the back one. Participant's input will be shown in the rectangular box below the string pair. (B) Time sequence of a single trial in evaluation stage. Participants choose the preferred string by click either one of the two buttons at the bottom of the screen.

Unbeknownst to the participants, in half of the trials, the string pair comprises a GV string and a GK string; and in the other half, it comprises a GD string and a GK string. The position of GK (upper or lower) was randomly determined at the onset of each trial.

\subsection{Data Analysis}

Two preference scores (PSs) were calculated for each individual participant based on their choices in the evaluation stage. PS-GD corresponds to the proportion of the 24 GD-GK trials in which GD was favored by the participant, while PS-GV corresponds to the proportion of the $24 \mathrm{GV}-\mathrm{GK}$ trials in which GV was favored by the participant. Hence, a PS-GD of over 0.5 means that GD strings were preferred by the participant over GK strings. In a similar vein, a PS-GV of over 0.5 means that GV strings were preferred by the participant over GK strings.

\section{Results}

A one $t$-test was conducted on the PS-GD scores to determine whether their mean was significantly different from 0.5 , the expected value if the choice was made by chance. The result shows that the mean of PS-GD is significantly larger than $0.5(M=0.58$, $S D=0.15), t(25)=2.9, p=0.008$.

A one t-test was conducted on the PS-GV scores to determine whether their mean was significantly different from 0.5 , the expected value if the choice was made by chance. The result shows that the mean of PS-GV is significantly larger than $(M=$ $0.42, S D=0.18$ ) which is significantly smaller than $0.5, t(25)=-2.4, p=0.025$. 


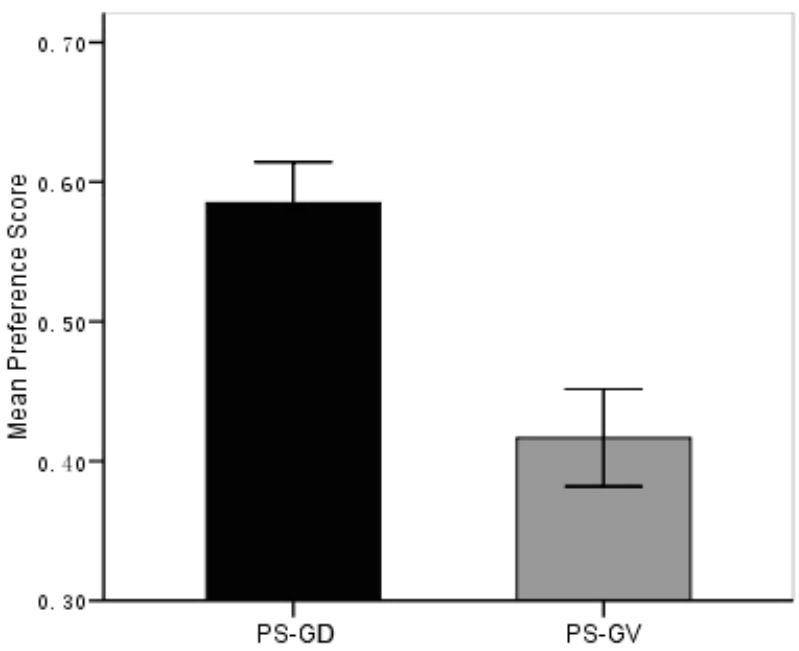

Fig. 2. Mean preference score for GD strings (PS-GD) and mean preference score for GV (PS$\mathrm{GV})$. Vertical bars indicate plus or minus one standard error of mean.

Taken together (Fig. 2), the results from the present study indicate that if the unseen, emotionally-bland stimuli belong to the same family as the previously attended stimuli (i.e. GD strings), they were more likely to become the preferred ones when pitted against other unseen, emotionally-bland stimuli based on a novel rule (i.e. GK strings). However, if the unseen, emotionally-bland stimuli belong to the same family as the previously inhibited stimuli (i.e. GV strings), they were less likely to be favored by participants when pitted against other unseen, emotionally-bland stimuli based on a novel rule (i.e. GK strings).

\section{Discussion}

In this experiment, we measure the affective evaluation of a meaningless character string conforming to the same rule as either the attended strings or inhibited strings in a prior implicit learning task. A previous artificial grammar learning study using a similar design provided evidence that participants could acquire abstract rules underlying both the attended strings and the inhibited ones simultaneously [17]. Therefore, assuming participants extracted the rules of both GD strings (attended) and GV strings (inhibited) unconsciously, the novel and important finding of current experiment is that even previously unseen stimuli can be subject to the devaluating effect of attentional inhibition as long as these stimuli share certain commonalities (e.g. underlying grammars in the present study) with those previously inhibited stimuli and these commonalties have been successfully learned by participants. In other words, we showed that the "dislike" toward previously inhibited stimuli can be spread to previously unseen stimuli of the same family. This new discovery about the interaction between three major systems - affective system, attentional system, and information acquisition system in the human brains-will no doubt have far-reaching 
implication for the design and evaluation of both affective system and affective agents.

Note that in this study, participants showed greater liking toward GD (attended rule) strings than GK (novel rules) strings. Such result could easily be explained by the perceptual fluency theories without recourse to the devaluation-by-inhibition hypothesis. Perceptual fluency theories assert that prior experience with a given stimuli makes future process of the same stimuli less taxing, hence leads to positive affect during subsequent encounter [20]. However, the result concerning GV (inhibited rule) strings stands in stark contrast with the prediction from perceptual fluency theories. In the present study, GV strings, though inhibited by attentional system, were exposed 120 (15 trials $\times 8$ blocks) times more than GK strings, providing a great opportunity for stimuli of this grammar to develop fluency, hence would have led to positive affect in the evaluation stage compared to string of novel grammar. Yet, they were still devalued significantly, which is more in line with distracter devaluation effect [6].

Two potential problems might undermine the validity of the conclusion drawn from present study. First, the assumption that participants acquired both the attended and the inhibited rules may not hold true. Though an above-chance preference score (mean PS-GD = 0.58) for the GD (attended rule) strings may indicate that participants have obtained knowledge about GD [19], there is little support from available literature suggesting that a below-chance preference score can also be taken as the evidence of the acquisition of abstract rule by participants. Therefore, it is a priority for future studies to investigate whether people is capable of extracting rule from inhibited strings under the same experimental condition. Second, since all the participants in the current experiment were required to attend to GD strings while inhibiting GV ones, the preference scores obtained may simply have been caused by this systematic bias. Though in theory, the intrinsic affective values of the strings used in this experiment should not differ as a function of their underlying grammars, future study is nevertheless needed to address this possible confounding effect.

Acknowledgments. This research was supported by grants from 973 Program of Chinese Ministry of Science and Technology (\#2006CB303101), and the National Natural Science Foundation of China (\#60433030).

\section{References}

1. Anderson, A.K., Wais, P.E., Gabrieli, J.D.E.: Emotion Enhances Remembrance of Neutral Events Past. PNAS 103, 1599-1604 (2006)

2. Bechara, A.: The Role of Emotion in Decision-making: Evidence from Neurological Patients with Orbitofrontal Damage. Brain and Cognition. 55, 30-40 (2004)

3. Bush, G., Luu, P., Posner, M.I.: Cognitive and Emotional Influences in Anterior Cingulate Cortex. Trends in Cognitive Sciences. 4, 215-222 (2000)

4. Cacioppo, J.T., Gardner, W.L.: Emotion. Annual Review of Psychology, pp. 191-192 (1999)

5. Dienes, Z., Altmann, G.T.M., Kwan, L., Goode, A.: Unconscious Knowledge of artificial grammars is applied strategically. Journal of Experimental Psychology: Learning, Memory, and Cognition 21, 1322-1338 (1995) 
6. Fenske, M.J., Raymond, J.E.: Affective Influences of Selective Attention. Current Directions in Psychological Science. 15, 312-316 (2006)

7. Fenske, M.J., Raymond, J.E., Kessler, K., Westoby, N., Tipper, S.P.: Research Report Attentional Inhibition Has Social-Emotional Consequences for Unfamiliar Faces. Psychological Science. 16, 753 (2005)

8. Hao, F., Zhang, H., Fu, X.: Modulation of Attention by Faces Expressing Emotion: Evidence from Visual Marking. In: Tao, J., Tan, T., Picard, R.W. (eds.) ACII 2005. LNCS, vol. 3784, Springer, Heidelberg (2005)

9. Knowlton, B.J., Squire, L.R.: Artificial Grammar Learning Depends on Implicit Acquisition of Both Rule-based and exemplar-based information. Journal of Experimental Psychology. 1150, 169-181 (1996)

10. McCabe, S.B., Gotlib, I.H.: Selective Attention and Clinical Depression: Performance on a Deployment-of-attention Task. J Abnorm Psychol. 104, 241-245 (1995)

11. Raymond, J.E., Fenske, M.J., Westoby, N.: Emotional Devaluation of Distracting Patterns and Faces: A Consequence of Attentional Inhibition During Visual Search? Journal of Experimental Psychology: Human Perception and Performance 31, 1404-1415 (2005)

12. Reber, A.S.: Implicit learning of synthetic languages. Journal of Verbal Learning and Verbal Behavior. 6, 855-863 (1967)

13. Rowe, G., Hirsh, J.B., Anderson, A.K.: Positive Affect Increases the Breadth of Attentional selection. PNAS 104, 383-388 (2007)

14. Schneider, W., Eschman, A., Zuccolotto, A.: E-Prime User's Guide: Psychology Software Tools (2002)

15. Stadler, M.A., Frensch, P.A.: Handbook of implicit learning: Sage Publications. Sage Publications, Thousand Oaks, CA (1998)

16. Strongman, K.T.: The Psychology of Emotion: From Everyday Life to Theory, 5th edn. Wiley, Chichester (2003)

17. Tanaka, D., Kiyokawa, S., Yamada, A., Shigemasu, K.: Investigating the Role of Selective Attention in Implicit Learning Using Overlapping Letter Strings. Paper presented at the 28th Annual Conference of the Cognitive Science Society, Vancouver, British Columbia, Canada (2006)

18. Vokey, J.R., Brooks, L.R.: Salience of Item Knowledge in Learning Artificial Grammars. Journal of Experimental Psychology: Learning, Memory, and Cognition 18, 328-344 (1992)

19. Whittlesea, B.W., Wright, R.L.: Implicit (and explicit) learning: acting adaptively without knowing the consequences. Journal of Experimental Psychology: Learning, Memory, and Cognition. 23, 181-200 (1997)

20. Winkielman, P., Schwarz, N., Fazendeiro, T., Reber, R.: The Hedonic Marking of Processing Fluency: Implications for Evaluative Judgement. The psychology of Evaluation: Affective Processes in Cognition and Emotion, 189-117 (2003) 